\title{
基于 2-卤苯甲酰胺合成苯并杂环化合物的研究进展
}

\author{
杨 凯 $a, b$ \\ 刘美娟 $a$ \\ 张毓娜 $a$ \\ 占佳琦 $a$ \\ 邓璐璇 $a$ \\ 郑雪洁 $a$ 周永军 $a$ 汪朝阳 $*, a$ \\ ( $a$ 华南师范大学化学学院 教育部环境理论化学重点实验室 广州市生物医药分析化学重点实验室 广州 510006) \\ ( $b$ 赣南医学院药学院 江西赣州 341000)
}

\begin{abstract}
摘要 苯并稠杂环化合物具有广泛且重要的药理及生物活性, 在药物合成与研发领域有着重要的应用价值, 因而其合 成方法倍受关注. 由于存在多个官能团和反应位点，以 2-卤代苯甲酰胺为重要合成子合成喹唑啉酮、异喹啉酮、异吲 哚酮和苯并异噻唑酮等苯并杂环化合物取得了巨大进展. 鉴于此, 根据产物结构的不同, 概述近 10 年来以 2-卤苯甲酰 胺为起始原料合成苯并杂环化合物的研究进展，并对相关的反应机理进行了阐述.
\end{abstract}

关键词 2-卤苯甲酰胺; 金属催化; 串联反应; 环化反应; 苯并杂环化合物; 反应机理

\section{Progress in the Synthesis of Benzoheterocycles from 2-Halobenzamides}

\author{
Yang, Kai ${ }^{a, b} \quad$ Liu, Meijuan $^{a} \quad$ Zhang, Yu'na $^{a} \quad$ Zhan, Jiaqi $^{a} \quad$ Deng, Luxuan $^{a}$ \\ Zheng, Xuejie $^{a} \quad$ Zhou, Yongjun $^{a} \quad$ Wang, Zhaoyang ${ }^{*, a}$ \\ ( ${ }^{a}$ Key Laboratory of Theoretical Chemistry of Environment, Ministry of Education, Guangzhou Key Laboratory of Analytical \\ Chemistry for Biomedicine, School of Chemistry, South China Normal University, Guangzhou 510006) \\ ( ${ }^{b}$ College of Pharmacy, Gannan Medical University, Ganzhou, Jiangxi 341000)
}

\begin{abstract}
Owing to important pharmacological and biological activities of benzoheterocyclic compounds, their exploration for efficient synthesis methods in drug field is of great value and wide application. Due to the existence of multiple functional groups and reaction sites, great progress has been made in the synthesis of quinazolinones, isoquinolinones, isoindolinones, benzisothiazolones and other benzoheterocyclic compounds using 2-halobenzamides as important synthons. In view of this, according to the different structures of the products, the research progress in the synthesis of benzoheterocyclic compounds with 2-halobenzamides as the starting material in recent 10 years is summarized, and the related reaction mechanism is expounded.

Keywords 2-halobenzamide; metal catalysis; domino reaction; cyclization reaction; benzoheterocyclic compound; reaction mechanism
\end{abstract}

含氮苯并稠杂环化合物是数目庞大的一类有机化 合物, 在精细化工、药物化学、农业化学和材料化学等 领域有着极其广泛的应用 ${ }^{[1]}$. 其中，喹唑啉酮[2]、异喹啉

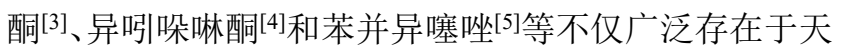
然产物中，且因其具有显著的生物和药理活性，常被应 用于药物分子的研究开发(图 1). 因此, 利用结构简单且 易得的起始原料, 通过高效、便捷的合成方法来构筑苯
并稠杂环化合物, 已经成为有机合成化学中一个重要的 研究领域 ${ }^{[6]}$.

在 2-卤苯甲酰胺这个简单的分子中，同时存在多种 官能团, 如苯环、卤原子、羰基和氨基, 且酰胺基与卤 原子处于苯环上相邻位置. 鉴于这种独特的结构特点, 其可先经过 Sonogashira 偶联 ${ }^{[7]} 、$ Ullmann ${ }^{[8]}$ 或 BuchwaldHartwig 偶联[9]等反应形成碳-碳键和碳一杂键, 再串联环

\footnotetext{
* Corresponding author. E-mail: wangzy@scnu.edu.cn

Received January 26, 2021; revised February 20, 2021; published online March 4, 2021.

Project supported by the Guangzhou Science and Technology Project Scientific Special (No. 201607010251), the Guangdong Provincial Science and Technology Project (No. 2017A010103016), the Scientific Research Fund of Jiangxi Provincial Education Department (No. GJJ201504), the Gannan Medical University (No. YB201903) and the Undergraduates Innovation Project of Guangdong Province (No. S202010574059).

广州市科技计划科学研究专项(No. 201607010251)、广东省科技计划(No. 2017A010103016)、江西省教育厅科学技术研究(No. GJJ201504)、赣南医学 院(No. YB201903)和广东省大学生创新创业训练计划(No. S202010574059)资助项目.
} 
化 ${ }^{[10]}$, 从而实现各类含氮苯并稠杂环化合物的合成 ${ }^{[11]}$.

目前, 2-卤苯甲酰胺常作为重要的合成子用于构建 苯并杂环化合物及药物分子, 在过去的十多年间已取得 了令人瞩目的进展. 国内外已有一些基于其他合成子的 杂环化合物合成方法的文献综述报道 ${ }^{[12]}$, 但是基于 2-卤 苯甲酰胺为底物的合成方法文献综述未见报道. 因此, 本文根据产物结构的不同, 概述近 10 年来以 2-卤苯甲 酰胺为起始原料合成苯并杂环化合物的研究进展.<smiles>[R]OC(=O)OCc1ccc(N(Cc2ccc3c(=O)[nH]c(C)nc3c2)N(C(=O)O)c2ccc(C(=O)NC(CCC(=O)O)C(=O)O)s2)o1</smiles><smiles>COc1cc2ccn(C)c(=O)c2cc1O</smiles><smiles>CC(C)(C)OCCCCCCCC1C(=O)C2C3C=CC(C3)C2C(=O)C1C[C@@H]1CCCCC1N1CCN(c2nsc3ccccc23)CC1</smiles><smiles>COc1ccc2c(c1OC)C(=O)N/C2=C\c1ccccc1CCN</smiles>

图 1 具有苯并杂环化合物的天然产物及药物分子

Figure 1 Natural products and drug molecules with benzoheterocyclic compounds

\section{1 喹唑啉酮类化合物的合成}

作为苯并六元杂环化合物的热门分子, 喹唑啉酮是 构成许多天然产物及药物分子的核心单元 ${ }^{[13]}$. 2-卤苯甲 酰胺可与茮胺、酰胺或腈等物质反应，实现喹唑啉酮类 衍生物的合成(Scheme 1).

\subsection{2-卤苯甲酰胺与胺反应合成喹唑啉酮类化合物}

付华课题组 ${ }^{[14]}$ 以 2-卤代苯甲酰胺和芳基甲胺作为 起始原料, 以溴化亚铜为催化剂, 空气为氧化剂, 利用 串联反应，一步合成了喹唑啉酮类衍生物 (Scheme 2). 该反应体系适用于碘代底物、溴代底物和具有硝基的氯 代底物，也适用于 2-噻吩甲胺和 2-吡喃甲胺等芳基甲胺 底物，产率为 $43 \% \sim 86 \%$.

随着研究的深入, 越来越多的铜盐催化体系可以实 现此类转化. 例如, 在 $\mathrm{CuSO}_{4} \cdot 5 \mathrm{H}_{2} \mathrm{O}$ 或 $\mathrm{CuI}$ 催化下, 由 2-碘苯甲酰胺衍生物和苄胺、烯丙胺或肉桂胺等反应, 可以 $35 \% \sim 82 \%$ 的产率合成 2 -苯基喹唑啉酮类衍生

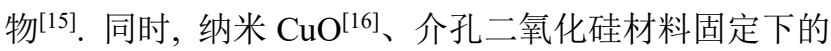
$L$-脯氨酸铜(I)复合物 ${ }^{[17]}$ 和铜锰尖晶石氧化物 ${ }^{[18]}$ 等各类 铜盐催化体系亦被开发并应用于该反应，且这些催化体 系都具有多次回收重复催化效果.

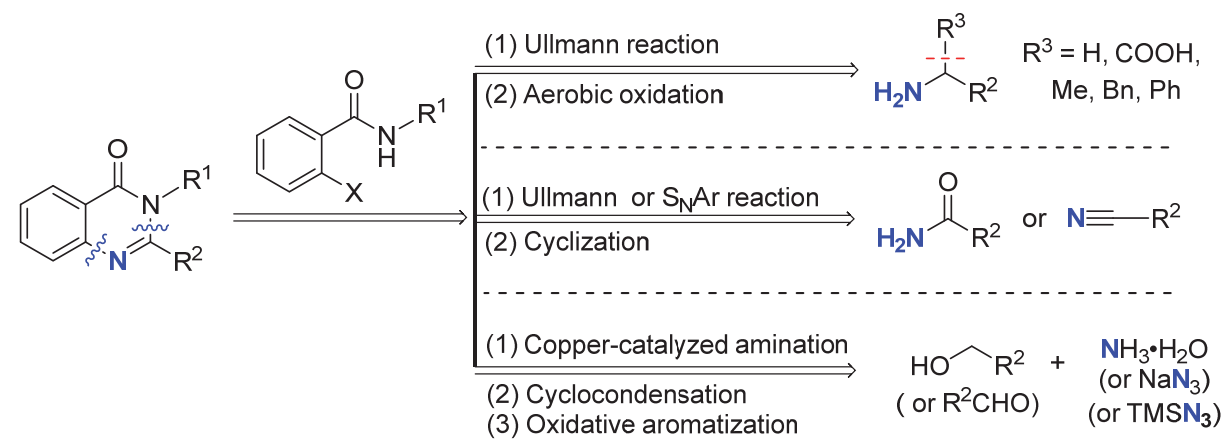

图式 1 2-卤苯甲酰胺合成喹唑啉酮分子的逆合成分析

Scheme 1 Retrosynthetic analysis for the synthesis of quinazolinones from 2-halobenzamides

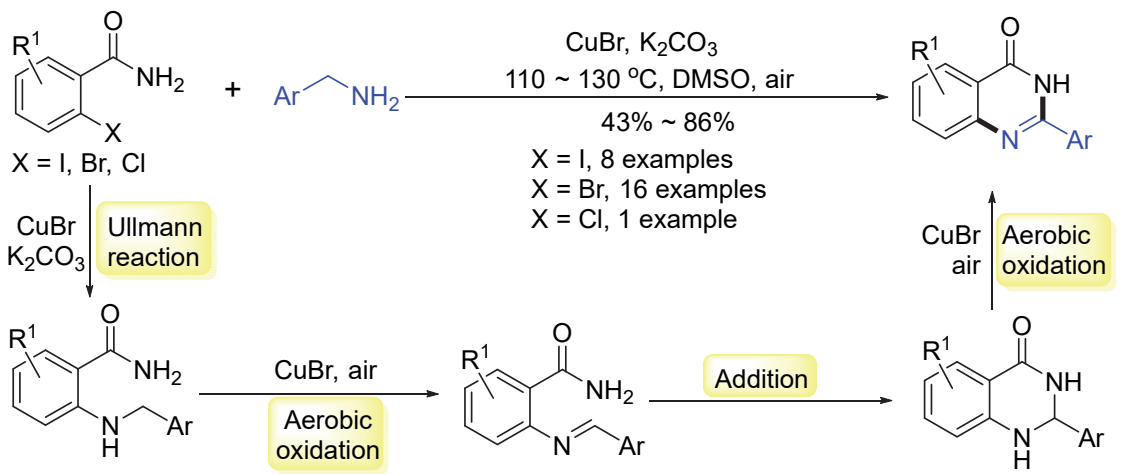

图式 2 铜催化合成喹唑啉酮类化合物

Scheme 2 Copper catalyzed synthesis of quinazolinone derivatives 
对于一些 $\alpha$-取代的苠胺, 通过分子内 $\mathrm{C}-\mathrm{C}$ 键的断 裂, 也可为喹唑啉酮化合物的制备提供一种新的方法. 唐亚林课题组 ${ }^{[19]}$ 报道, 空气氛围中, 在 $\mathrm{CuBr} / \mathrm{K}_{2} \mathrm{CO}_{3}$ 催 化下，以 2-卤苯甲酰胺与 $\alpha$ 位含有甲基、苯基和茮基等 取代基的苄胺为原料, 无需添加任何配体即可实现 2-芳 基喹唑啉酮的合成(Scheme 3). 若在 $\mathrm{CuBr}_{2} / \mathrm{K}_{2} \mathrm{CO}_{3}$ 催化 体系下, 反应可在氩气氛围下完成 ${ }^{[20]}$.

除了苄胺类化合物能与 2-卤苯甲酰胺生成喹唑啉 酮外, $\alpha$-氨基酸也能与之发生反应, 其中经历了 Ullmann 偶联、脱羧、氧化和分子内加成等过程 (Scheme 4), 产 率为 $40 \% \sim 72 \%$ [21]. 类似的反应在多相催化剂氧化石墨 烯(GO)与 $\mathrm{Fe}_{3} \mathrm{O}_{4}-\mathrm{CuI}$ 混合作用下也能发生, 且该复合催 化剂有较高的催化效率, 四次催化循环后产率基本不 变 ${ }^{[22]}$.

一些特殊结构的烯胺和环胺等，也可与 2-卤苯甲酰 胺发生成环反应 ${ }^{[23-24]}$. 例如, 在 $\mathrm{CuI} / \mathrm{Cs}_{2} \mathrm{CO}_{3}$ 催化体系中, 对 2-碘苯甲酰胺和 $Z$-烯胺酮的反应, $Z$-烯胺酮也充当配 体, 反应先后经过 Ullmann $\mathrm{C}-\mathrm{N}$ 偶联、Michael addition 和 retro-Mannich 等过程，产率为 20\% 78\% (Scheme 5, a); 若反应底物是 $E$-烯胺酮，则需额外加入配体 $L$-脯氨 酸, 如在 $\mathrm{CuI} / L$-proline 催化下合成喹唑啉酮类化合物, 产率为 $14 \% \sim 81 \%$ (Scheme $5, b)^{[25]}$.

不仅如此, 一些苠胺型的环仲胺类底物, 也可实现 类似的成环反应. 例如, 在氮气氛围下 $\mathrm{CuI}$ 可催化 2-卤 苯甲酰胺和 1,2,3,4-四氢异喹啉衍生物发生分子间偶联, 再串联氧化反应及分子内环化，从而合成四氢异喹啉并 $[2,1-a]$ 喹唑啉酮衍生物(Eq. 1) ${ }^{[26]}$.

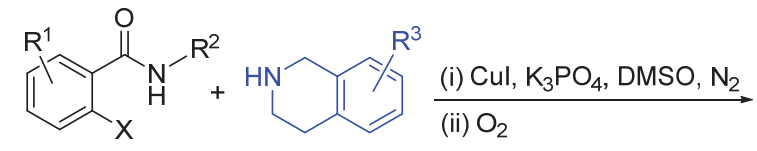

$\mathrm{X}=\mathrm{Cl}, \mathrm{Br}, \mathrm{I} \quad \mathrm{R}^{3}=\mathrm{H}$, alkyl, OMe, $\mathrm{Cl}$

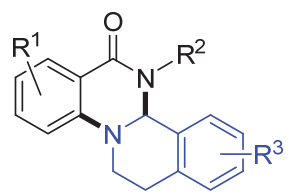

31 examples, $41 \% \sim 89 \%$

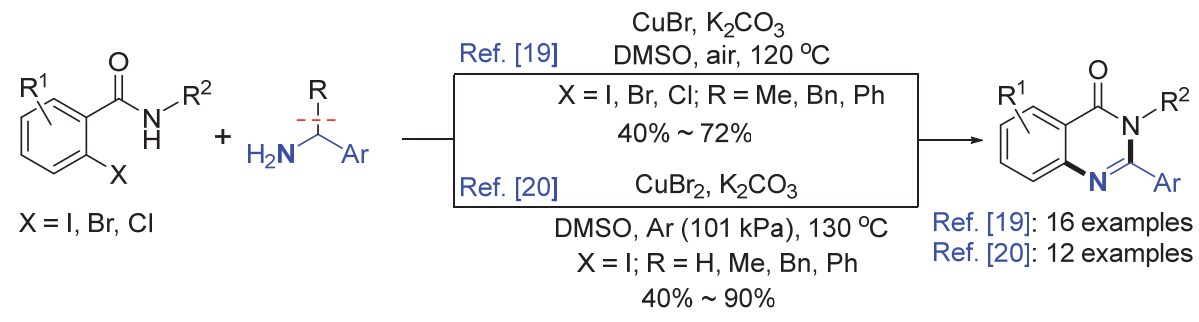

图式 3 2-卤苯甲酰胺和芐胺合成 2-芳基喹唑啉酮

Scheme 3 2-Arylquinazolinones were synthesized from 2-halobenzamides and benzylamines

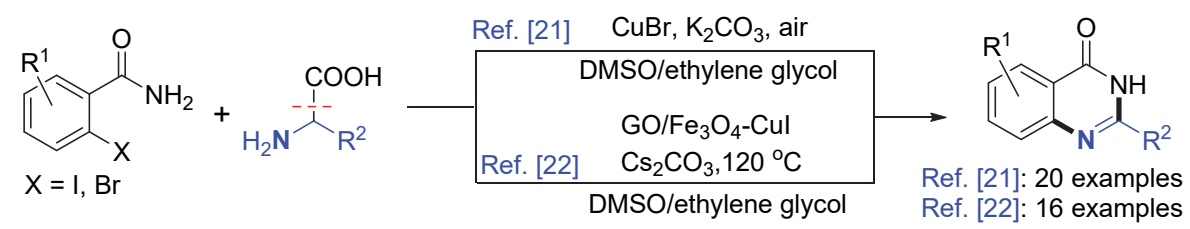

图式 4 2-卤苯甲酰胺和 $\alpha$-氨基酸合成喹唑啉酮

Scheme 4 Synthesis of quinazolinones from 2-halobenzamides and $\alpha$-amino acids

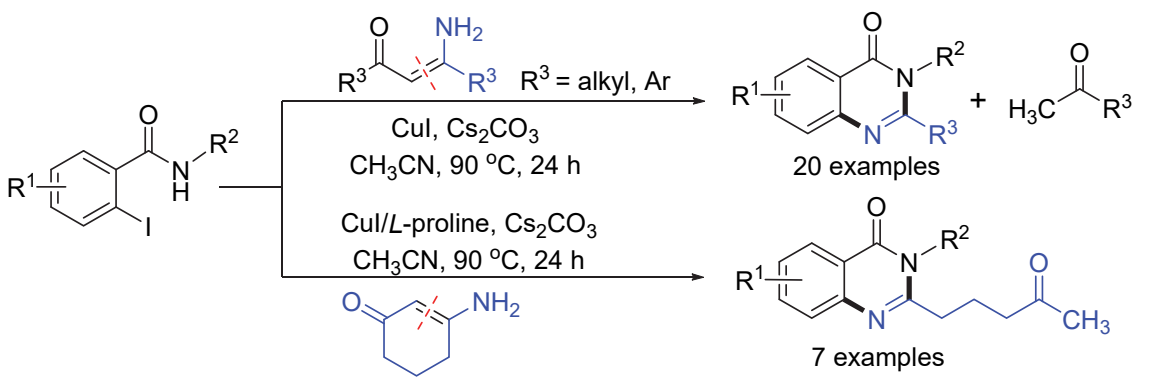

图式 5 2-卤苯甲酰胺和烯胺酮合成喹唑啉酮

Scheme 5 Synthesis of quinazolinones from 2-halobenzamides and enaminones 


\subsection{2-卤苯甲酰胺与酰胺、腈等反应合成喹唑啉酮类化} 合物

除了胺类化合物, 酰胺化合物也可与 2-卤苯甲酰胺 反应合成喹唑啉酮类衍生物. 马大为课题组 ${ }^{[27]}$ 报道了 以 2-溴苯甲酰胺和甲酰胺为原料, 在 $\mathrm{CuI} / 4$-羟基- $L$-脯氨 酸的催化下，合成喹唑啉酮，产率达 69\% 95\%. 若反 应底物为脂肪族和芳香族酰胺, 则需先分离偶联产物, 再在六甲基二硅氮烷( $\mathrm{HMDS}$ )和 $\mathrm{ZnCl}_{2}$ 作用下进行反应, 其产率为 $60 \% \sim 96 \%$ (Eq. 2).

具有循环重复使用效果的 $\mathrm{CuI}$ 纳米粒子(CuI-NP), 亦可用于催化 2-溴/氯苯甲酰胺和芳酰胺发生分子间的 环化反应，产率为 $72 \% \sim 81 \%$ (Scheme 6, Method A $)^{[28]}$. 甚至对于底物为 2-氟苯甲酰胺的反应, 无需金属催化, 在 $\mathrm{Cs}_{2} \mathrm{CO}_{3}$ 作用下, 即可与酰胺经过 $\mathrm{S}_{\mathrm{N}} \mathrm{Ar}$ 反应串联分子 内环化，其产率可达 81\% (Scheme 6, Method B) ${ }^{[29]}$.

若以乙醇为溶剂, 微波辅助, 在 $\mathrm{CuI} / \mathrm{Cs}_{2} \mathrm{CO}_{3}$ 催化
下, 2-碘/溴代苯甲酰胺会先与乙醇发生 $\mathrm{C}-\mathrm{O}$ 偶联反应, 然后再与另一分子 2-碘/溴代苯甲酰胺底物发生分子间 的缩合得到 3-(2-乙氧苯基)喹唑啉酮类化合物. 由于在 该反应中醇既当溶剂又为反应物, 故存在一定量的乙醇 偶联副产物(Eq. 3 $)^{[30]}$.

除了酰胺类化合物, 腈类分子在 $\mathrm{Cu}(\mathrm{OAc})_{2}$ 的催化 下，也可与 2-卤苯甲酰胺反应合成喹唑啉酮类化合物 (Eq. 4) ${ }^{[31]}$. 通过类似的成键顺序去连接 2-卤苯甲酰胺合 成子中的相同原子，异硫氰酸酯在 $\mathrm{CuI}$ 和 $N, N^{\prime}$-二甲基乙 二胺(DMEDA)催化下, 则可与 2-溴苯甲酰胺一步合成 2-硫代-2,3-二氢喹唑啉-4(1H)-酮(Eq. 5) ${ }^{[32]}$.

\subsection{2-卤苯甲酰胺与氨水或叠氮化合物等三组分反应 合成喹唑啉酮类化合物}

从结构简单、廉价易得的原料出发，利用多组分反 应合成目标分子，是合成化学的绿色化策略 ${ }^{[33]}$. 基于 2卤苯甲酰胺与醛、氨水/叠氮化合物等发生的三组分反
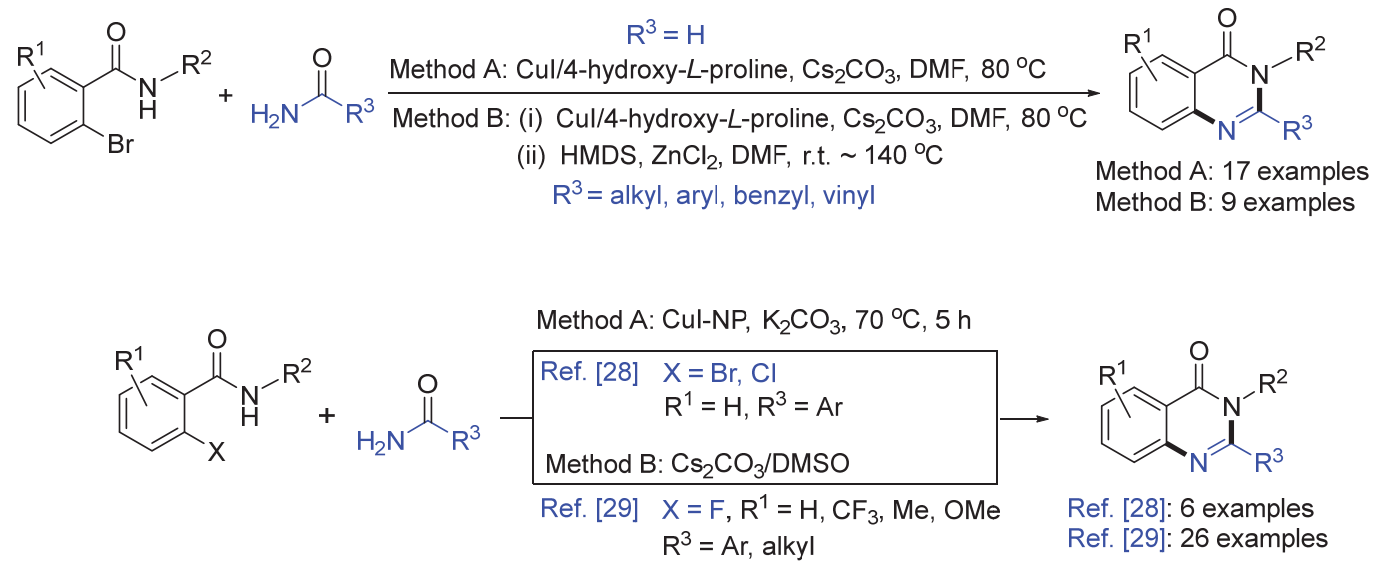

图式 6 2-卤苯甲酰胺和酰胺合成喹唑啉酮

Scheme 6 Synthesis of quinazolinones from 2-halobenzamides and amides
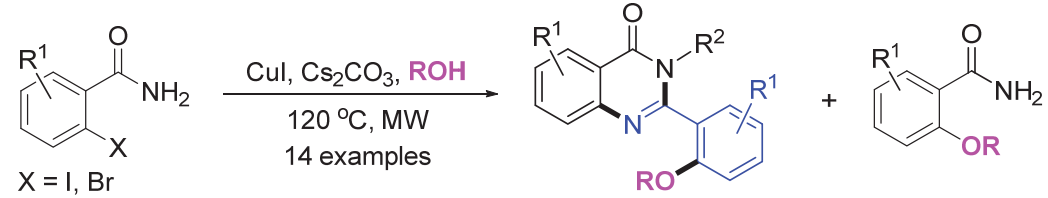

$\mathrm{R}^{1}=\mathrm{Br}, \mathrm{Cl}, \mathrm{Me}, \mathrm{OMe}$

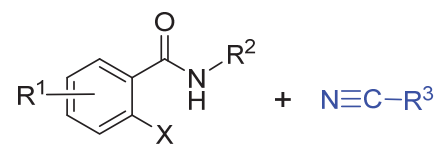

$\mathrm{X}=\mathrm{I}, \mathrm{Br} ; \mathrm{R}^{1}=\mathrm{Me}, \mathrm{OMe} \mathrm{R}^{3}=$ aryl, alkyl

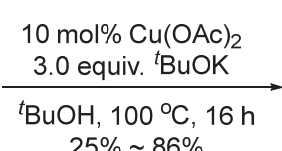

$25 \% \sim 86 \%$

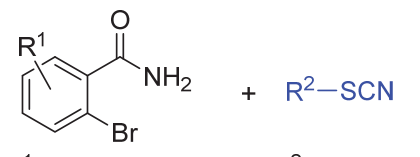

$\frac{10 \mathrm{~mol} \% \mathrm{Cul}, 20 \mathrm{~mol} \% \text { DMEDA }}{\mathrm{Cs}_{2} \mathrm{CO}_{3} \text {, toluene, } 120^{\circ} \mathrm{C}, 24 \mathrm{~h}}$ $62 \% \sim 90 \%$<smiles>[Y]c1nc2cc[R1]cc2c(=O)n1[R]</smiles>

20 examples<smiles>[R]#CCOC(=O)n1c(=S)[nH]c(=O)c2ccccc21</smiles> 
应，可合成喹唑啉酮类化合物 ${ }^{[34-35]}$. 例如, $\mathrm{CuBr} / L$-脯氨 酸催化下，2-溴苯甲酰胺与醛和氨水在空气中经过胺 化、缩合、环化及分子内氧化等串联反应过程, 可合成 喹唑啉酮类化合物(Eq. 6) ${ }^{[36]}$.
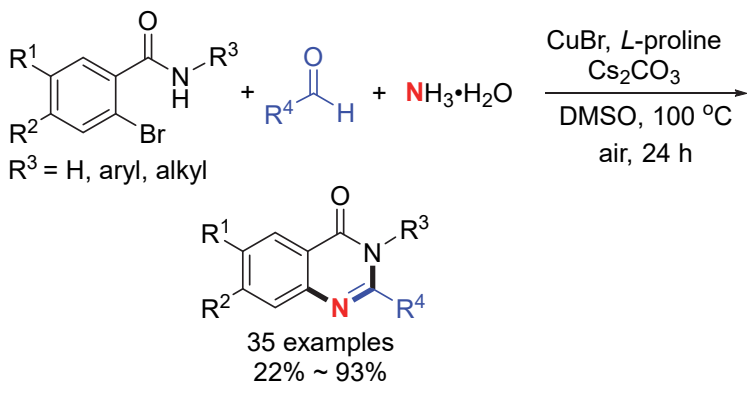

不仅如此, 若以叠氮三甲基硅烷 $\left(\mathrm{TMSN}_{3}\right)$ 作氮源, 2-溴苯甲酰胺也可与苯甲醛、苯甲醇或甲基芳烃反应， 经过氧化加成、还原胺化、苄基 $\mathrm{C}-\mathrm{H}$ 的分子间氧化氮 化、分子内环化和氧化脱氢等历程, 一锅法合成喹唑啉 酮类化合物(Eq. 7). 需要指出的是, 在该反应中, 若以 苯甲醛为原料不需要使用叔丁基过氧化氢(TBHP), 而 以苯甲醇、甲基芳烃为原料则需要 ${ }^{[37]}$.

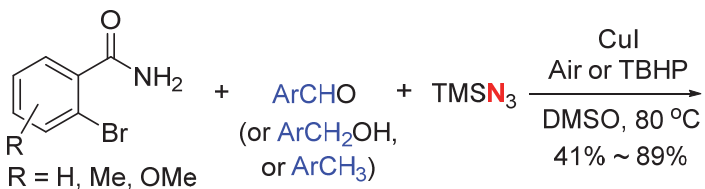

$$
\begin{aligned}
& \text { examples }
\end{aligned}
$$

叠氮化钠也可作为氮源, 应用于类似转化. 以 2-溴 苯甲酰胺、醛和叠氮化钠为原料, 在 $\mathrm{CuBr} / L$-脯氨酸催 化下，能以 $50 \%$ ～95\%的产率合成喹唑啉酮类化合物 (Scheme 7, Method A ${ }^{[38]}$. 若以芳香甲醇或 $N, N$-二甲基 乙酰胺为原料, 则需要添加额外的 2,2,6,6-四甲基
哌啶氧化物(TEMPO) $)^{[39]}$ 或 $\mathrm{TBHP}^{[40]}$ 为氧化剂(Scheme 7, Methods B, C).

\section{4 其他喹唑啉酮稠杂环化合物的合成}

2-卤苯甲酰胺还可和其他杂环化合物反应，合成喹 唑啉酮稠环化合物 ${ }^{[41]}$. 例如，以 2-卤苯甲酰胺衍生物和 咪唑类衍生物为起始原料, $\mathrm{CuI}$ 或 $\mathrm{CuBr}$ 为催化剂, $L$-脯 氨酸为配体, $\mathrm{Cs}_{2} \mathrm{CO}_{3}$ 为碱，二甲基亚砜(DMSO)为溶剂, $120{ }^{\circ} \mathrm{C}$ 下先后在氮气和氧气氛围中反应，即可合成咪唑 啉酮/苯并咪唑啉酮类化合物，产率为 $53 \%$ ～98\% (Eq. $8)^{[42]}$.

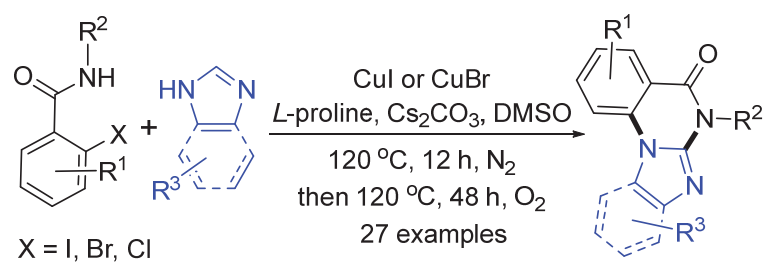

当反应物为吲哚-3-甲酸甲酯类化合物时，以 $\mathrm{CuBr}$ / $\mathrm{Cs}_{2} \mathrm{CO}_{3}$ 为催化体系, 空气氛围下在 DMSO 中回流反应, 即可合成吲哚 $[1,2-a$ ]喹唑啉酮类化合物，产率为 52\% $93 \%(\mathrm{Eq} .9)^{[43]}$. 需要指出的是, 该方法中吲哚底物中羧 酸甲酯部分为 Ullmann $N$-芳基化/2-酰胺化串联反应中 的活化基团. 利用类似策略，简单高效地合成各类稠杂 多环化合物，具有巨大的发展空间及研究前景.

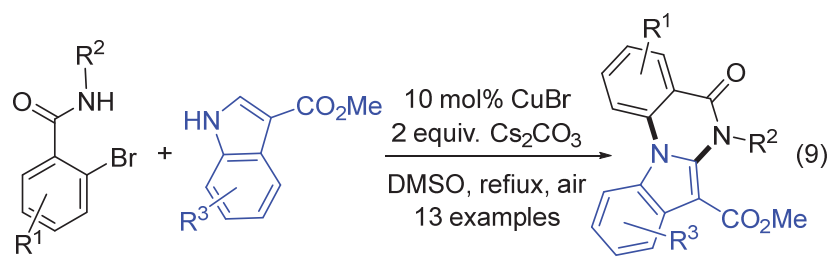

\section{2 异喹啉酮骨架化合物的合成}

异喹啉酮骨架常作为重要的结构单元，广泛存在于 天然产物、生物活性分子和药物中 [44].2-卤苯甲酰胺作 为原料砌块，可与炔、丙二腈或酮类等化合物反应合成 异喹啉酮衍生物(Scheme 8)

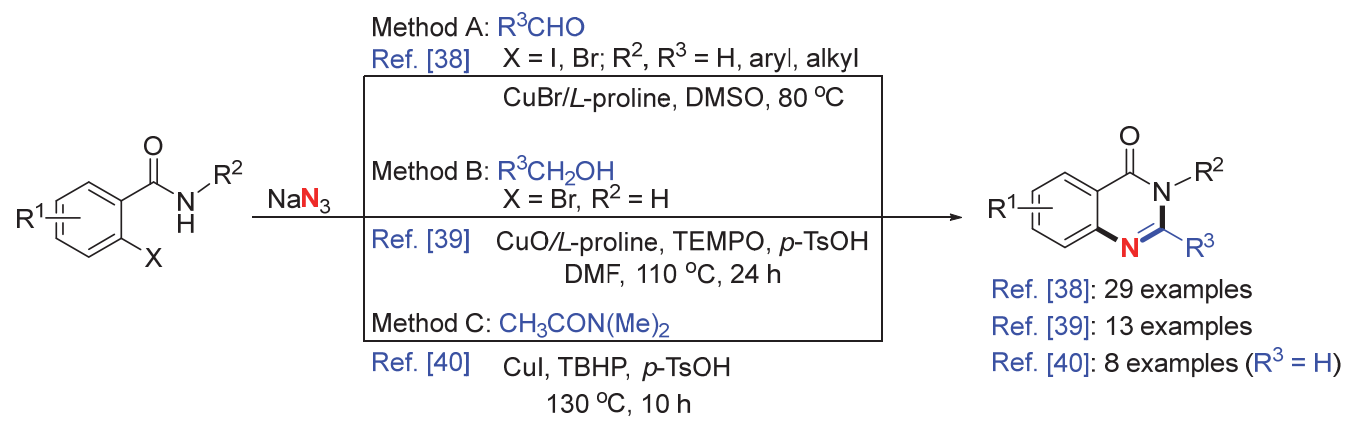

图式 7 2-卤苯甲酰胺和叠氮化钠合成喹唑啉酮

Scheme 7 Synthesis of quinazolinones from 2-halobenzamides and sodium azide 


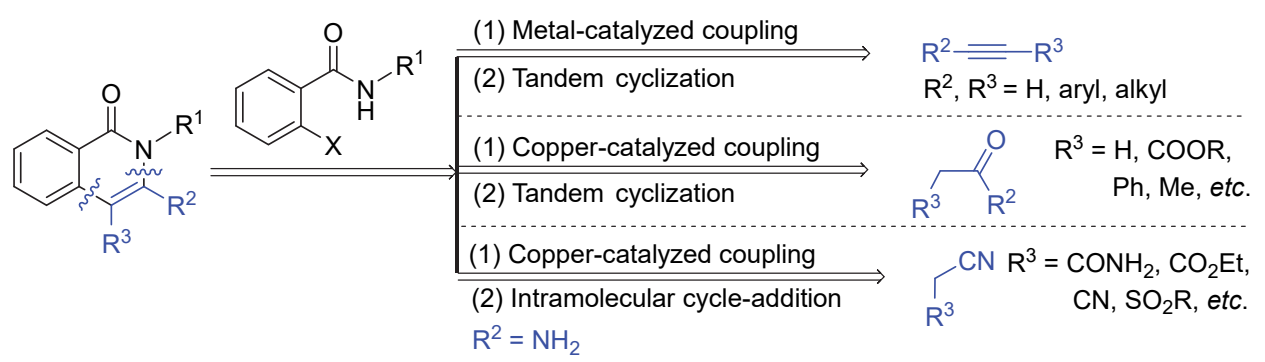

图式 8 2-卤苯甲酰胺合成异喹啉酮类分子的逆合成分析

Scheme 8 Retrosynthetic analysis for the synthesis of isoquinolinones from 2-halobenzamides

\subsection{2-卤苯甲酰胺与炔反应合成异喹啉酮类化合物}

作为最常见的 C(2)合成子, 炔可与 2-卤苯甲酰胺通 过金属 Ni 催化的偶联环化反应合成异喹啉酮类化合 物 ${ }^{45]}$. 郑建鸿课题组 ${ }^{[46]}$ 利用该方法, 以 $\mathrm{NiBr}_{2}$ (dppe)/Zn 为 催化体系, $\mathrm{Et}_{3} \mathrm{~N}$ 为碱, 完成了异喹啉酮衍生物的合成, 产率可达 63\% 92\% (Eq. 10). 该反应体系中锌粉为还 原剂, 起催化作用的为零价 Ni. 此方法已被成功应用于 天然产物 Oxyavicine 的全合成, 总产率高达 76\%. 值得 注意的是, 若底物为不对称的炔, 则可能存在位置异构 的产物 ${ }^{[45-46]}$.

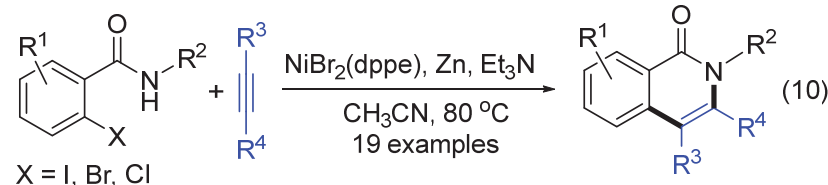

最近, Chatani 课题组 ${ }^{[4]}$ 利用零价的双-(1,5-环辛二 烯)镍为催化剂, 成功实现了由 2 -氟苯甲酰胺与对称炔 烃合成异喹啉酮衍生物的反应，产率为 $74 \% \sim 99 \%$. 控 制实验和密度泛函理论计算(DFT)结果表明，该反应机 制可能为 2-氟苯甲酰胺的 $\mathrm{NH}$ 去质子化后与 $\mathrm{Ni}$ 配位形 成 $\mathbf{B}$, 其 $\mathrm{C}-\mathrm{F}$ 键断裂氧化加成形成五元镍(II)杂环 $\mathbf{C}$, 然后炔烃的迁移插入形成中间体 $\mathbf{D}$, 最后还原消除得到 产物同时再生成 $\mathrm{Ni}(0)$ (Scheme 9).

不仅如此, 金属 Co 盐复合物也被应用于催化此类 反应. 最近, Perez-Temprano 课题组 ${ }^{[48]}$ 系统研究了一类 复合 $\mathrm{Cp} * \mathrm{Co}(\mathrm{III})$ 催化剂对各类邻位含有甲酰类导向基团 的芳基卤化物相关偶联反应的催化机制及应用，发现该 催化剂可实现 2-碘苯甲酰胺和二苯基乙炔的偶联环化 合成 3,4-二苯基异喹啉-1(2H)-酮, 产率为 66\% (Eq. 11).

事实上, 相对廉价的过渡金属 $\mathrm{Cu}$ 盐也可实现类似 转化. 以聚乙二醇 PEG-400 为溶剂, $\mathrm{Cu}(\mathrm{OAc})_{2} / \mathrm{Cs}_{2} \mathrm{CO}_{3}$ 催化下，2-碘苯甲酰胺可与末端炔反应合成异喹啉酮衍 生物, 产率为 $56 \% \sim 93 \%$ (Eq. 12) ${ }^{[49]}$. 需要指出的是, $\mathrm{Cu}(\mathrm{OAc})_{2}$ 大的空间位阻可能使反应发生 Sonogashira 偶

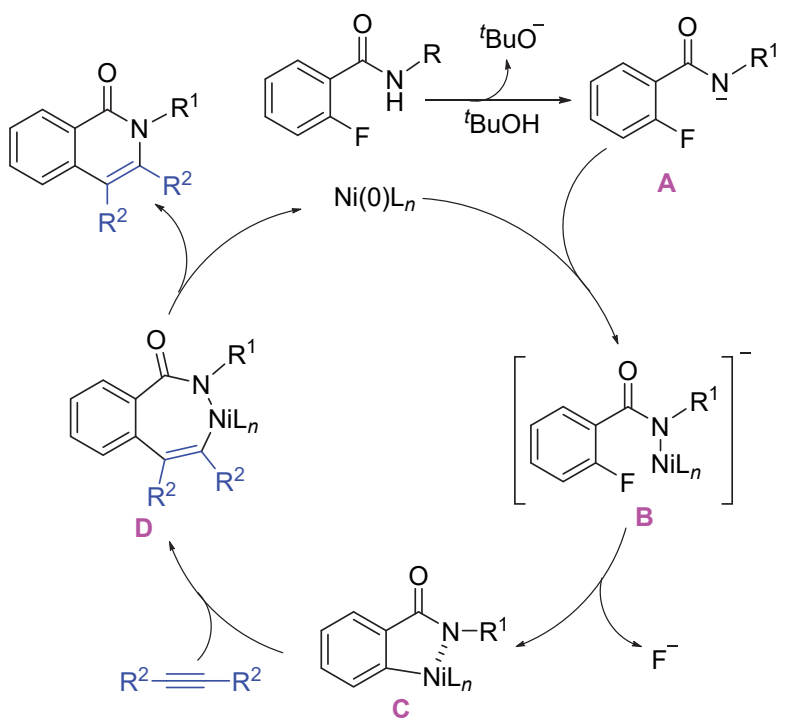

图式 $9 \mathrm{Ni}$ 催化 2-氟甲酰胺与炔的反应机理

Scheme 9 Reaction mechanism of $\mathrm{Ni}$ catalyzed 2-fluorobenzamides with alkynes

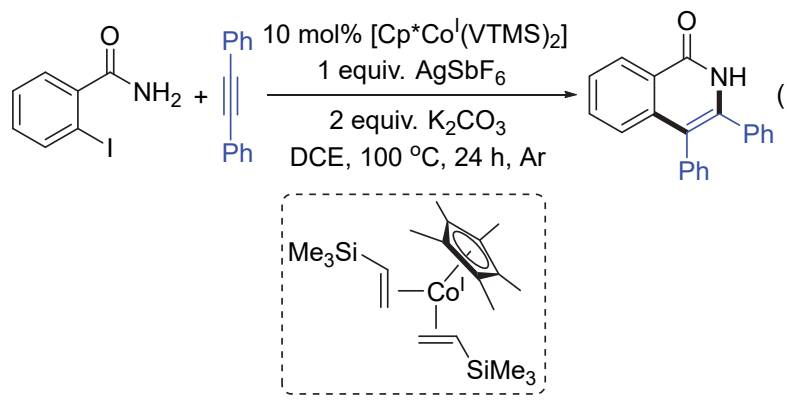

$\mathrm{Cp}^{*} \mathrm{Co}^{\prime}(\mathrm{VTMS})_{2}$

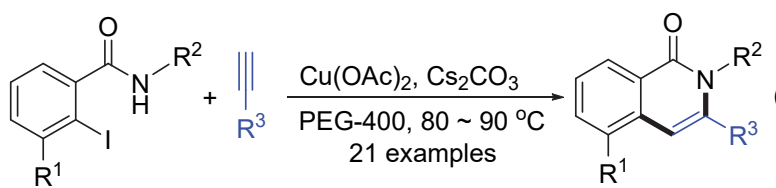

联，6-endo 型环化得到六元环产物，而不是常见的铜催 化五元环化产物一一异吲哚酮类化合物. 


\subsection{2-卤苯甲酰胺与 1,3-二酮、腈类等化合物合成异喹 啉酮类化合物}

利用 $\mathrm{Cu}$ 盐催化, 2-卤苯甲酰胺也可与存在活泼 $\alpha$ 氢的酮类等反应, 先通过脱卤化氢发生 $\mathrm{C}-\mathrm{C}$ 偶联, 再 经过分子内亲核加成消除(环化)过程，一步合成异喹啉 酮类化合物 ${ }^{[50]}$. 例如, 以 $\mathrm{CuI} / \mathrm{Cs}_{2} \mathrm{CO}_{3}$ 为催化体系, $\beta$-酮 酸酯可在 $80{ }^{\circ} \mathrm{C}$ 氮气氛围下发生偶联环化反应, 产率达 $32 \% \sim 86 \%$ (Eq. 13) $)^{[51]}$.

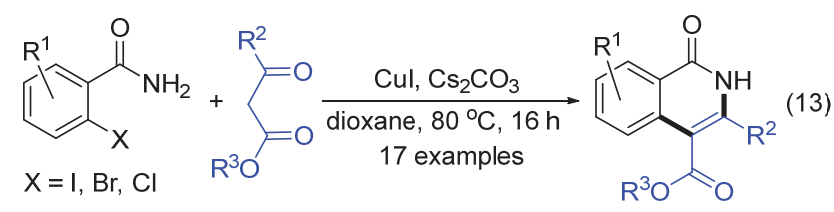

很明显, 若一些环状结构的特殊二羰基化合物参与 该反应，则可合成多环稠杂环化合物. 例如，1,3-狮二酮 类化合物可与 2-碘苯甲酰胺在 $\mathrm{CuCl}_{2} / \mathrm{Cs}_{2} \mathrm{CO}_{3}$ 催化下, 以 $49 \% \sim 85 \%$ 的产率合成茚异喹啉酮衍生物(Eq. 14). 该方 法可以用于一步法合成一系列临床活性拓扑异构酶 I 抑 制剂 NSC314622, LMP-400 和 LMP-776 ${ }^{[52]}$.<smiles>[R]c1ccc2c(c1)C(=O)[C]([R]NC(=O)c1c([R])cccc1I)C2=O</smiles>

不仅如此, 利用 $\mathrm{CuBr} / \mathrm{Cs}_{2} \mathrm{CO}_{3}$ 催化, 2-碘(溴)苯甲酰 胺也可与苯乙酮、杂环芳甲酮或环酮(环己酮和环戊酮 等)等各种含 $\alpha$-氢的酮类化合物反应, 合成各种多取代 的异喹啉酮类化合物，产率为 33\% 94\% (Eq. 15) ${ }^{[53]}$.

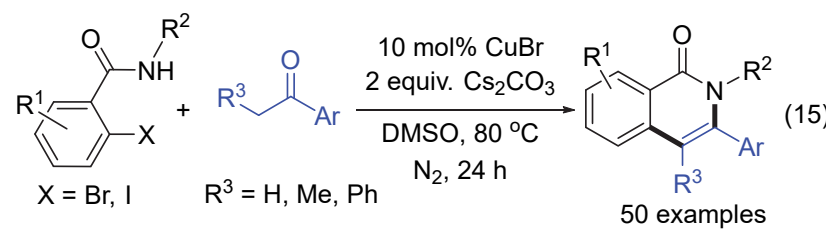

若利用光辅助催化, 在无铜盐催化下, 2-碘苯甲酰 胺也可与芳乙酮类化合物通过 $\mathrm{S}_{\mathrm{RN}} 1$ 反应历程, 实现 3取代异喹啉酮类化合物的合成，产率可高达 $91 \%{ }^{[54]}$. 相 对于常规的 $\mathrm{Cu}$ 盐催化, 该方法不需要金属催化, 具有 反应条件温和及绿色等优点. 目前利用光催化实现类似 转化的报道较少, 故存在巨大的发展潜力.

若 2-卤苯甲酰胺与含活泼亚甲基腈类物质反应，则 可合成 3-氨基取代的异喹啉酮类衍生物 ${ }^{[55]}$. 例如，以 2碘苯甲酰胺与丙二腈等为起始原料, $\mathrm{Cu}(\mathrm{OAc})_{2} \cdot \mathrm{H}_{2} \mathrm{O}$ / $\mathrm{K}_{2} \mathrm{CO}_{3}$ 为催化剂体系, 可在 $90{ }^{\circ} \mathrm{C}$ 氮气氛围下发生偶联
环化, 产率为 $35 \% \sim 99 \%$ (Eq. 16) ${ }^{[56]}$.<smiles>[R][R]([H])C(C#N)NC(=O)c1c(I)cccc1I</smiles>

以一些结构特殊的 $N$-取代 2-卤代苯甲酰胺为原料, 利用腈转化得到的氨基继续反应，还可实现一些含异喹 啉酮结构的稠杂环化合物合成. 付华课题组 ${ }^{[57]}$ 巧妙地 使用 2-溴- $N$-(2-卤代苯基)苯甲酰胺为原料，使其与 2-㲵 乙酸烷基酯或丙二腈反应，一步构建了双环合成苯并咪 唑异喹诺酮类化合物，产率为 $63 \%$ ～91\% (Eq. 17).

$$
\text { (N) }
$$

不仅如此，当反应物为有氨基酸酯结构的 2 -溴代苯 甲酰胺时，则可和腈类化合物合成含有氨基酸残基的含 异喹啉酮结构的稠杂环化合物，产率 $61 \%$ 85\% (Eq. $18)^{[58]}$.

$$
\text { (20 examples }
$$

利用这种腈转化得到的氨基继续反应的稠杂环合 成策略，也可开发出有后续反应基团的腈类化合物，如 2-碘苯乙氰类，使其与 2 -碘苯甲酰胺反应，在 $\mathrm{CuCl} /$ 脯氨 酸催化下，能以 $74 \%$ \% $94 \%$ 的产率合成吲哚并异喹啉酮 类衍生物(Eq. 19) ${ }^{[59]}$. 因此，选择不同的底物可构建连 接方式不同的含异喹啉酮结构的稠杂环，该合成策略值 得借鉴.

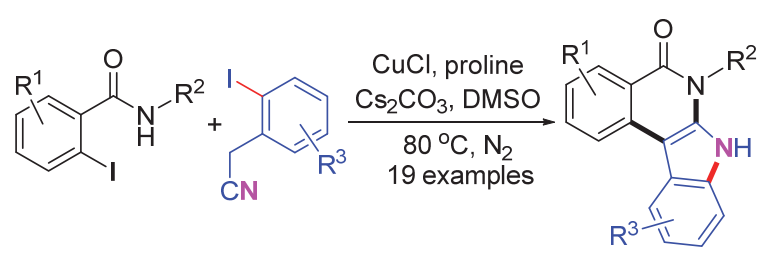




\subsection{2 -卤苯甲酰胺自身分子间或分子内环化合成菲啶 酮类化合物}

菲啶酮是一类含异喹啉酮骨架的稠环化合物, 常具 有抗菌、抗疮疾和抗肿瘤等生物活性, 在合成及药物化 学中得到广泛应用 ${ }^{600}$. 研究表明, 2-卤苯甲酰胺可通过 分子内 ${ }^{[6]}$ 或分子间 ${ }^{[62]}$ 环化反应实现菲啶酮的合成.

Chaudhary 课题组 ${ }^{[63]}$ 以 1-(2-差帮乙基)-哌嗪为有机小 分子催化剂(Orgcat.), 在叔丁醇钾促进下, 以 $N$-苯基-2溴代苯甲酰胺为原料, 通过自由基历程发生分子内环 化，可合成菲啶酮类化合物(Eq. 20). 由于其自由基环化 过程中, 存在 6-exo/endo 或 5-exo 二种成环方式, 故当 $N$-取代苯环上存在取代基时可能有取代基位置异构产 物 ${ }^{[63-64] \text {. }}$

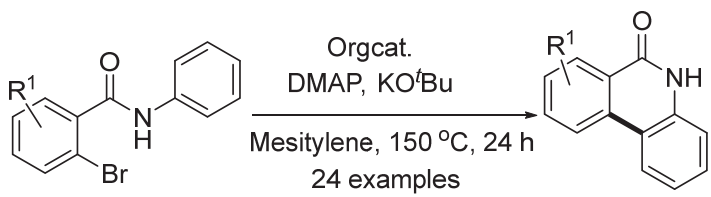

利用金属 Pd 催化，也可实现类似的分子内环化 ${ }^{[65]}$. 余洛汀课题组 ${ }^{[66]}$ 以 $\mathrm{Pd}\left(t-\mathrm{Bu}_{3} \mathrm{P}\right)_{2}$ 为催化剂, 叔丁氧羰基 (Boc)保护的 $N$-苯基邻卤苯甲酰胺可发生分子内环化和 脱羧而实现菲啶酮的一锅法合成，产率为 $37 \%$ ～95\% (Eq. 21).

以 2-卤代苯甲酰胺为原料, 亦可通过自身的二分子 间环化反应合成菲啶酮类化合物 ${ }^{[67]}$. 例如，在 $\mathrm{PdCl}_{2}$ $(\mathrm{PhCN})_{2} / \mathrm{CsF}$ 作用下, 2 -溴(氯)苯甲酰胺可先脱卤发生自

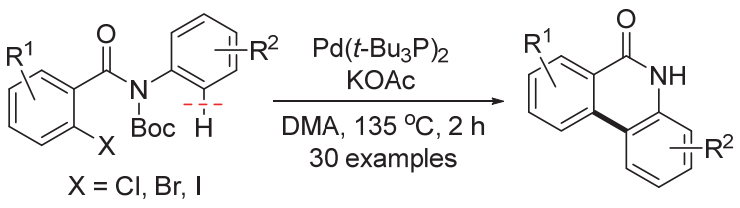

身 $\mathrm{C}-\mathrm{C}$ 偶联, 然后经过消除异氰酸酯串联环化, 一锅 合成菲啶酮类化合物(Scheme 10) ${ }^{[68]}$. 当然，若以微波辐 射辅助, $\mathrm{Pd}(\mathrm{OAc})_{2} / \mathrm{PPh}_{3} / \mathrm{NaOH}$ 为催化体系, 在 DMSO 中 反应，邻溴(碘)苯甲酰胺也可在短时间内快速实现类似 转 化 ${ }^{[69]}$.

有趣的是, 在 $\left[\mathrm{PdCl}_{2}\left(\mathrm{PPh}_{3}\right)_{2}\right] / \mathrm{K}_{2} \mathrm{CO}_{3}$ 催化体系中, 若 在不同溶剂进行反应，可选择性地得到不同菲啶酮类产 物. 溶剂为二甲基甲酰胺(DMF)时，得到的是常见的脱 酰胺基的产物; 而在 1,4-二氧六环中，则得到不脱酰胺 基的产物(Scheme 11). 这可能与不同溶剂对反应所用的 碱的溶解度不同有关 ${ }^{[70]}$.

\section{3 异吲哚酮的合成}

2-卤苯甲酰胺也可作为重要合成子，用于合成苯并 五元杂环化合物 ${ }^{[71-72]}$. 其中，在 $\mathrm{Cu}$ 盐 ${ }^{[73]}$ 及 $\mathrm{Pd}$ 盐 ${ }^{[74]}$ 等金 属催化下，2-卤苯甲酰胺与炔类化合物反应合成异吲哚 酮类化合物最为常见. 异吲哚酮类化合物是许多天然产 物或药物分子的核心结构，具有抗病毒、抗肿瘤、抗艾 滋病病毒(HIV)、抗焦虑和治疗糖尿病等潜在生物活性 [75]. 因此, 异吲哚酮类化合物的合成引起了研究者的广 泛关注 ${ }^{[76]}$.

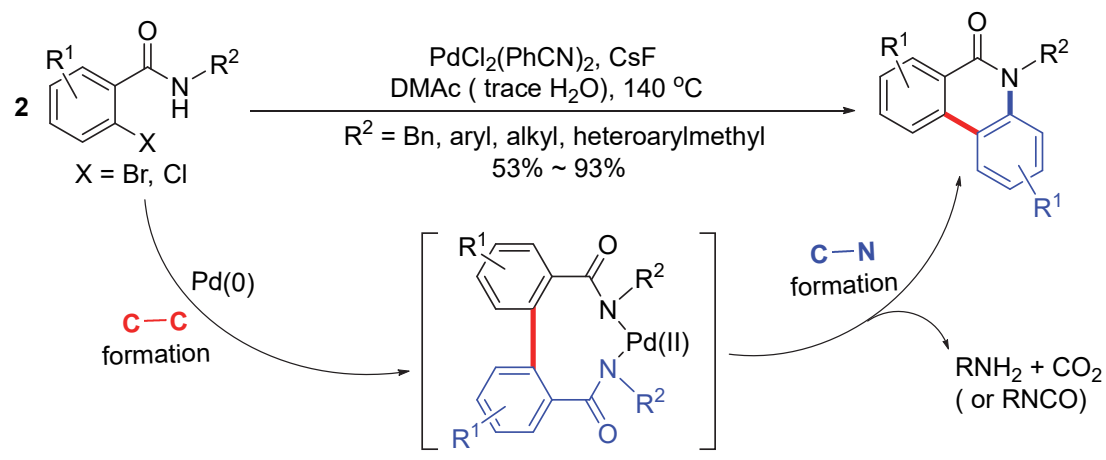

图式 10 钯催化邻卤苯甲酰胺合成菲啶酮

Scheme 10 Palladium-catalyzed synthesis of phenanthridinones from $o$-halobenzamides
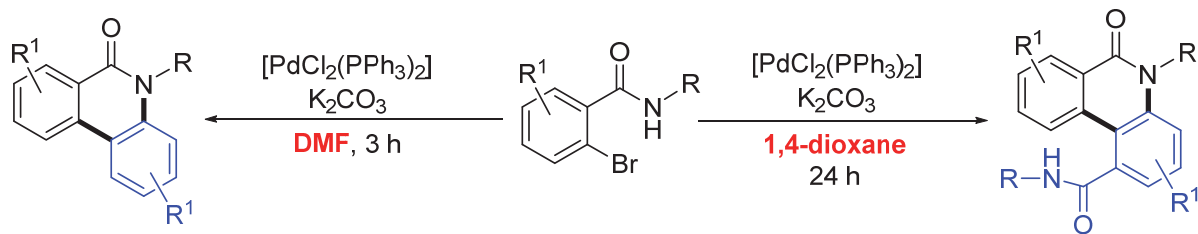

图式 11 钯催化邻溴苯甲酰胺的偶联反应

Scheme 11 Pd-Catalyzed coupling of $o$-bromobenzamides 
马大为课题组 ${ }^{[77]}$ 以 2-溴苯甲酰胺与末端炔为原料, 在 $\mathrm{CuI} / L$-脯氨酸催化下, 通过 Sonogashira 型偶联和分子 内 5-exo 型环化历程，一步实现了 3-亚甲基异吲哚-1-酮 的合成, 产率为 $55 \% \sim 93 \%$ (Scheme 12). 该反应主要得 到为 $Z$ 型产物, 仅当底物中存在硝基等某些特殊取代基 时主要为 $E$ 型产物. 目前, 该方法已被用于生物碱 lennoxamine 的合成. 随后, $\mathrm{Cu}(\mathrm{OAc})_{2} \cdot \mathrm{H}_{2} \mathrm{O} / 2,2^{\prime}$-联咪唑 ${ }^{[78]}$ 、 $\mathrm{Cu}(\mathrm{OAc})_{2} \cdot \mathrm{H}_{2} \mathrm{O} / 1,5$-二氮杂二环 $[5.4 .0]$ 十一烯-5 (DBU) ${ }^{[79]}$ 和络合物 $\left[\mathrm{Cu}(\mathrm{DMAP})_{4} \mathrm{I}\right] \mathrm{I}^{[80]}$ 等各类铜盐催化体系亦被开 发应用于此类转化, 且得到的都为 $Z$ 型产物.

除末端炔烃外，一些特殊结构的内炔也可与 2-卤苯 甲酰胺反应实现异吲哚-1-酮类化合物的合成 ${ }^{[81]}$. 例如, 以炔酸为炔源, 在 $\mathrm{CuI} / 1,10-\mathrm{Phen}$ 催化下, 经过脱羧交叉 偶联和串联 5-exo 环化, 即可合成 $Z$ 型异吲哚-1-酮类化 合物(Eq. 22 ${ }^{[82]}$. 该方法可避免 Sonogashira 反应中竞争 性二炔副产物的生成.

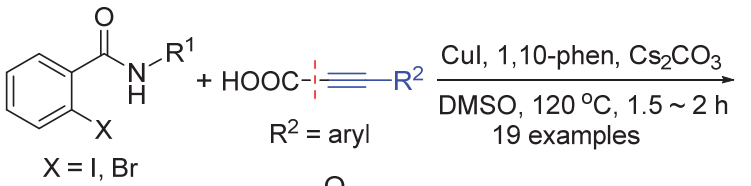

$$
\begin{aligned}
& \mathrm{R}^{2}
\end{aligned}
$$

除了常规的二组分反应, 利用一锅法串联反应, 可 在合成异吲哚啉-1-酮的基础上进一步衍生化 ${ }^{[83]}$. 例如, 以 2-卤苯甲酰胺、末端炔烃和 1-取代吲哚为原料, 在水 性胶束介质中, 通过三组分反应, 可合成 3-吲哚基异吲 哚啉-1-酮类衍生物(Eq. 23 ${ }^{[84]}$.

相比之下, 相对昂贵的 Pd 盐催化卤代苯甲酰胺和 末端炔烃合成异吲哚啉-1-酮的报道较为少见 ${ }^{[85]}$. 例如, 在微波条件下, 以 $\mathrm{Pd}(\mathrm{MeCN})_{2} \mathrm{Cl}_{2} / 1,1^{\prime}$-联荎- $2,2^{\prime}$-双二苯 膦(BINAP) 作为催化体系, 大多主要得到 $Z$ 型产物, 只

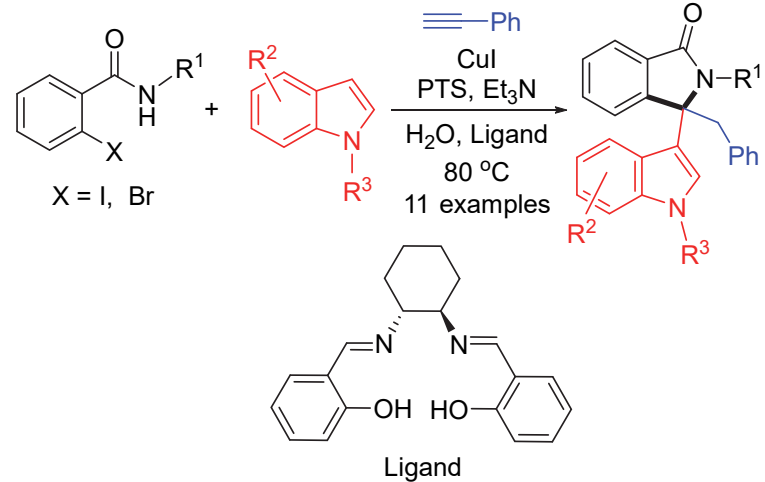

个别产物中存在一定比例 $E$ 型产物(Eq. 24) ${ }^{[86]}$.

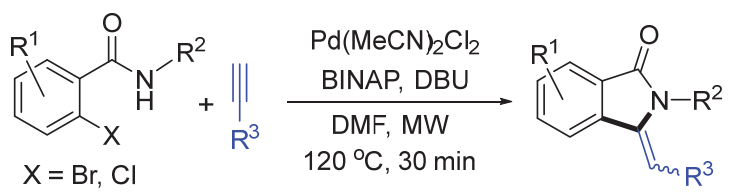

\section{4 苯并[d]异噻(硒)唑酮的合成}

以 2-卤苯甲酰胺为底物, 也能实现含硫(硒)杂环化 合物的合成 ${ }^{[87]}$. 其中, 苯并 $[d]$ 异噻(硒)唑酮类化合物因 具有显著的抗氧化功能 ${ }^{[88]}$, 其合成已成为研究热点 ${ }^{[89]}$. 通常, 2-卤苯甲酰胺与单质硫(硒)、二硒化锂、 KSCN $(\mathrm{KSeCN})$ 等反应，可合成苯并异噻(硒)唑-3(2H)-酮类化 合物.

$\mathrm{Cu}$ 盐催化卤代芳烃(烯烃)的 $\mathrm{C}-\mathrm{S} 、 \mathrm{C}-\mathrm{Se}$ 偶联反 应是有机合成中的常见方法 ${ }^{[00]}$, 以硫单质或硒单质与 2卤代苯甲酰胺反应合成含硫或硒杂环化合物时也不例 外 ${ }^{[91]}$. Kumar 课题组 ${ }^{[92]}$ 以 2-卤代苯甲酰胺和硒单质为原 料, 利用 $\mathrm{CuI} / 1,10-\mathrm{Phen}$ 催化, 可合成系列苯异硒唑3(2H)-酮类化合物，产率为 47\% 96\% (Scheme 13). 若 把原料硒换成为硫单质参与反应，则可合成苯并异噻 唑-3(2H)-酮类化合物，产率为 $40 \% \sim 95 \%{ }^{[93]}$.

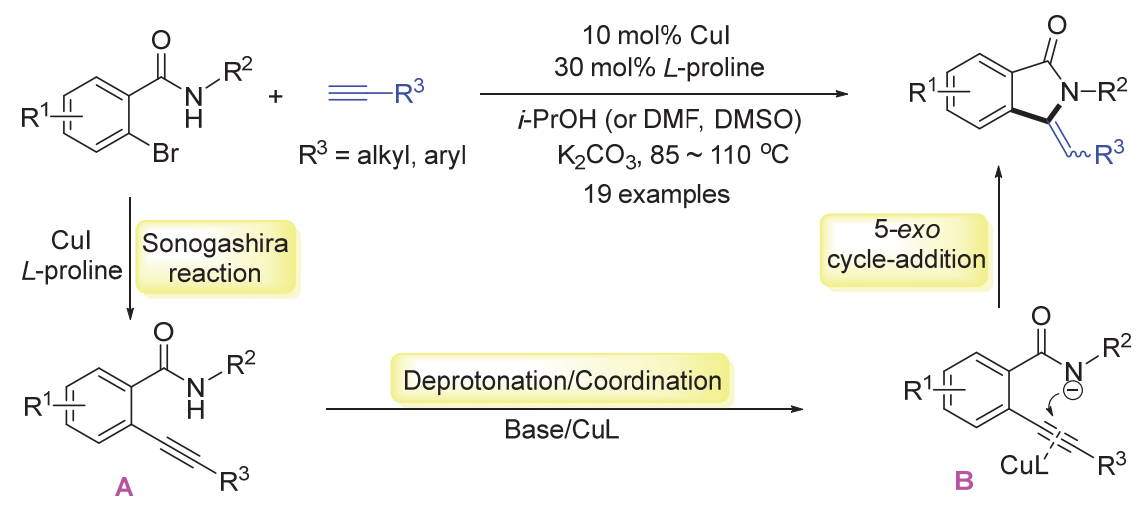

图式 $12 \mathrm{CuI} / L$-脯氨酸催化的 2-溴苯甲酰胺和末端炔烃的串联反应

Scheme $12 \mathrm{CuI} / L$-proline catalyzed domino reaction of 2-bromobenzamide and terminal alkynes 


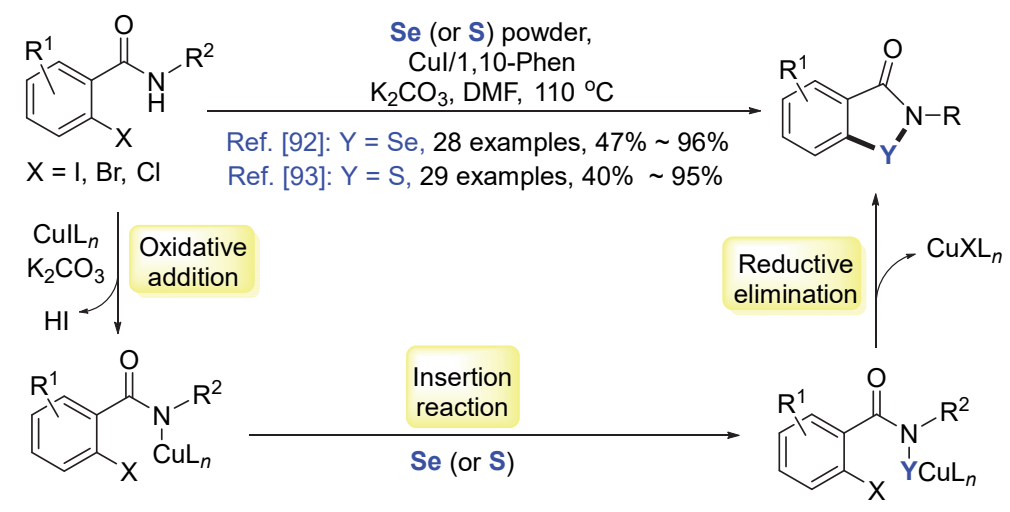

图式 13 苯并异硒(噻)唑酮的制备

Scheme 13 Preparation of benzoisothiazolones

若把硒单质制备为二硒化锂, 使其与 2-碘(溴)- $N$-苯 甲酰胺反应, 则不需要使用金属 $\mathrm{Cu}$ 盐催化. 在氩气氛 围下 $110{ }^{\circ} \mathrm{C}$ 反应, 可直接合成苯异硒唑-3(2H)-酮类化 合物, 产率为 $59 \% \sim 98 \%$ (Eq. 25) $)^{[94]}$.

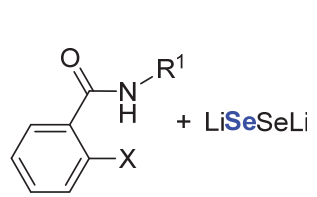

$\mathrm{X}=\mathrm{I}, \mathrm{Br}$ $\stackrel{(1) \mathrm{DMF}, 110^{\circ} \mathrm{C}, \mathrm{Ar}}{(2) \mathrm{H}_{2} \mathrm{O}}$
$\mathrm{R}^{1}=$ alkyl and aryl

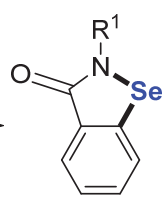

15 examples
(25)

$\mathrm{KSCN}(\mathrm{KSeCN})$ 也常作为硫(硒)源应用于有机合成 研究中 ${ }^{[95-96]}$. 在 CuI/1,10-Phen/DABCO (1,4-二氮杂二环 [2.2.2]辛烷)催化促进下，2-溴苯甲酰胺与硫氰酸钾可先 发生 $\mathrm{C}-\mathrm{S}$ 偶联, 形成芳基硫氰酸酯, 再消去㲵基经过
分子内亲核取代历程即可合成苯并异噻唑-3(2H)-酮类 化合物(Scheme 14) ${ }^{[97]}$. 类似地, 若以 $\mathrm{KSeCN}$ 为硒源, 在 $\mathrm{CuI} / 1,10-\mathrm{Phen} / \mathrm{Cs}_{2} \mathrm{CO}_{3}$ 催化下，可实现 2-碘苯甲酰胺对 苯异硒唑-3(2H)-酮的合 成 ${ }^{[98]}$.

\section{5 其他苯并杂环化合物的合成}

除常见的含氮、硫(硒)等苯并杂环化合物外, 2-卤苯 甲酰胺也能合成苯并啞嗪酮[99]和异香豆素 [100-101]等含氧 的苯并六元杂环化合物. 姚清发课题组 ${ }^{[102]}$ 以 2-卤苯甲 酰胺和 1,3-二羰基化合物为原料, $\mathrm{CuI}$ 为催化剂, $\mathrm{Cs}_{2} \mathrm{CO}_{3}$ 为碱, $\mathrm{DMSO}$ 为溶剂, 在 $100{ }^{\circ} \mathrm{C}$ 氮气环境下反应, 合成 了 3-取代-异香豆素类衍生物，产率为 56\% 98\% (Eq. 26).

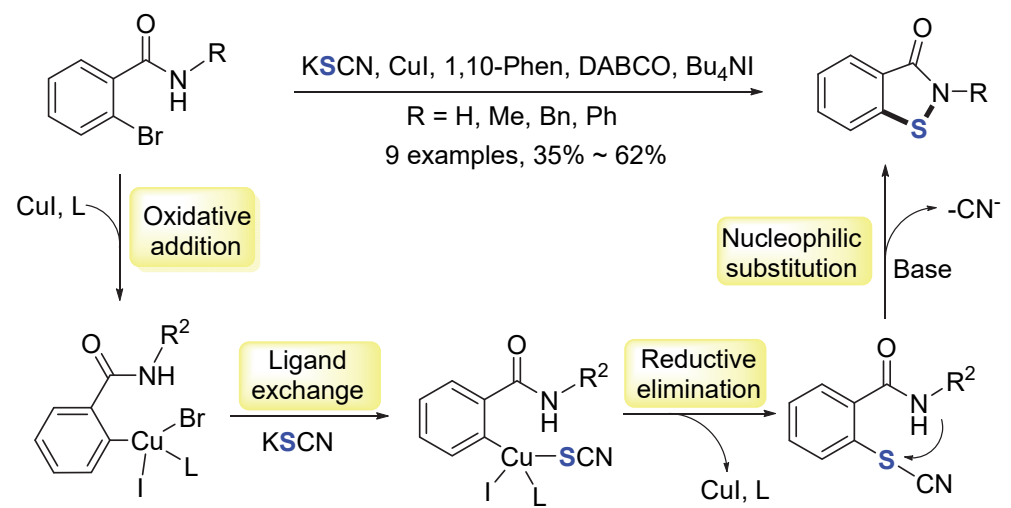

图式 14 2-溴苯甲酰胺和硫氰酸钾合成苯并异噻唑-3(2H)-酮

Scheme 14 Synthesis of benzisoselenazole-3(2H)-ones from 2-bromobenzoamides and potassium thiocyanate<smiles>[R]NC(=O)c1c([X])cccc1CC([R])=O</smiles>

$\mathrm{X}=\mathrm{I}, \mathrm{Br}, \mathrm{Cl}$<smiles>[R]NC(=O)CC</smiles>

$\mathrm{R}^{3}=$ alkyl and aryl 
除此之外, 2-卤苯甲酰胺还能用于含氮苯并七元杂 环化合物的合成 ${ }^{[103]}$. 例如, 以 2-卤苯甲酰胺衍生物和 2-碘苯胺衍生物为起始原料, 在 $\mathrm{CuI} / \mathrm{K}_{2} \mathrm{CO}_{3}$ 作用下, 通 过分子间的 Ullmann $\mathrm{C}-\mathrm{N}$ 偶联和分子内的 $\mathrm{C}-\mathrm{N}$ 酰胺 化过程, 则可合成二苯并二氮杂蒎酮类化合物, 产率为 $44 \% \sim 83 \%$ (Eq. 27 ${ }^{[104]}$.

$$
\text { (35 examples }
$$

除邻卤苯胺类底物外, 2-卤苯甲酰胺还可与邻卤酚 类底物反应, 实现含氧、氮的苯并七元杂环化合物的合 成 ${ }^{[105-106]}$. 例如, 以 2-碘苯甲酰胺和 2-溴苯酚为起始原 料, $\mathrm{CuI}$ 为催化剂, 二苯甲酰基甲烷为配体, $\mathrm{K}_{3} \mathrm{PO}_{4}$ 为碱, $\mathrm{DMF}$ 为溶剂, $120{ }^{\circ} \mathrm{C}$ 下反应, 可合成二苯并噁氮杂蒎酮 类化合物. 因该反应历程中会经历 Smiles 重排, 故有些 底物得到的为取代基位置异构产物(Scheme 15) ${ }^{[107]}$.

\section{6 总结与展望}

总而言之, 鉴于各类苯并杂环化合物在药物化学和 材料科学等领域的巨大应用潜力, 近年来以 2-卤苯甲酰 胺作为重要的合成子, 通过碳碳、碳杂键的构建, 各种 苯并杂环化合物的合成取得了长足的进展. 特别是基于 对过渡金属催化偶联、串联反应和多组分反应等的有效 应用，可根据不同结构特点的底物，有效地合成预期的 苯并杂环骨架化合物.

尽管如此, 该领域仍然存在许多挑战, 我们认为以 下两点值得关注: (1)目前基于 2-卤苯甲酰胺为底物对苯 并稠杂环化合物的合成，主要集中于传统加热条件下的 过渡金属催化偶联串联环化反应，而可见光催化合 成 ${ }^{[108]}$ 和电化学合成 ${ }^{[109]}$ 等绿色合成方法的研究应用较 少; (2)以 2-卤苯甲酰胺为底物的合成研究, 主要集中在 双环苯并杂环化合物的构建, 对于多环稠杂环化合物的 合成研究较少.

因此，由结构简单、廉价易得的原料，探寻篮选绿 色合成方法，简单、高效地合成苯并杂环化合物，仍然 是化学研究者继续努力的方向. 同时, 利用 2-卤苯甲酰 胺的结构特点, 开发多环稠杂环化合物具有巨大的研究 空间及应用潜力.

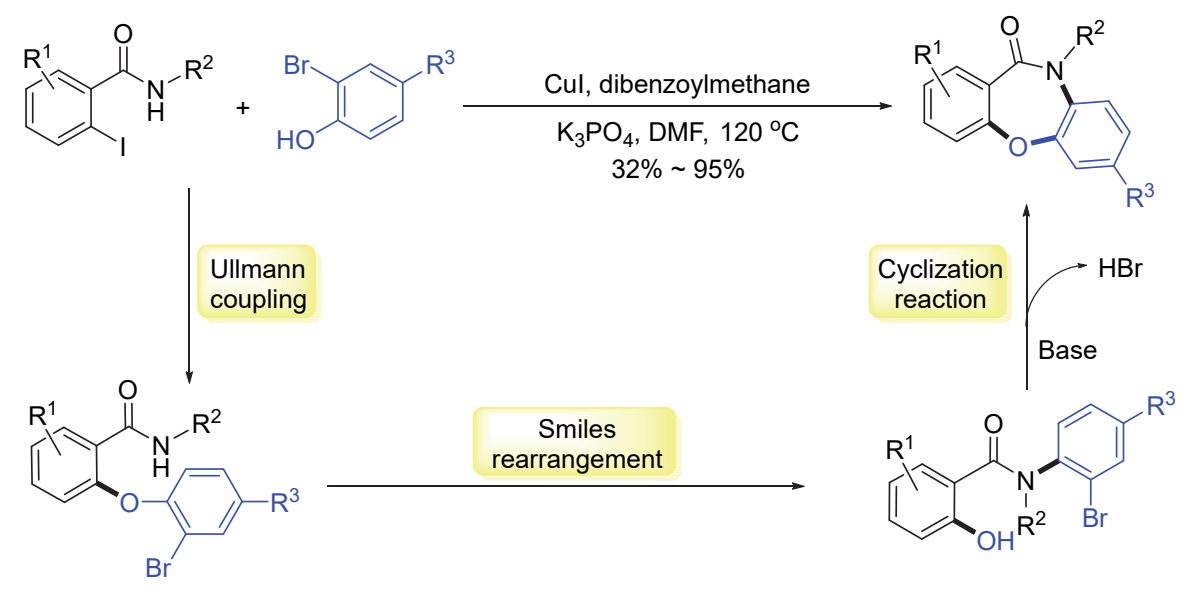

图式 15 二苯并啞氮杂蒎酮的合成

Scheme 15 Synthesis of dibenzoxazepinones

\section{References}

[1] Stepien, M.; Gonka, E.; Zyla, M.; Sprutta, N. Chem. Rev. 2017, $117,3479$.

[2] Zhang, X.-P.; Zhu, Y.-J.; Zhu, Y.-S.; Li, Z.-W.; Zhang, G.-S. Chin. J. Org. Chem. 2019, 39, 2392 (in Chinese).

(张晓鹏, 朱妍洁, 朱奕崧, 李政伟, 张贵生, 有机化学, 2019, 39, 2392.)

[3] (a) Doncheva, T.; Solongo, A.; Kostova, N.; Gerelt-Od, Y.; Selenge, D.; Philipov, S. Nat. Prod. Res. 2015, $29,853$.

(b) Yu, X.-Y.; Zhou, F.; Chen, J.-R.; Xiao, W.-J. Acta Chim. Sinica 2017, 75, 86 (in Chinese).

(余晓叶, 周帆, 陈加荣, 肖文精, 化学学报, 2017, 75, 86.)

[4] (a) Yao, T.-L.; Guo, Z.; Liang, X.-J.; Qi, L.-H. J. Org. Chem. 2018, 83,13370 . (b) Zhang, L.-B.; Wang, Z.-C.; Sun, S.-Z.; Ni, S.-F.; Wen, L.-R.; Li, M. Chin. J. Chem. 2021, 39, 903.

[5] Novick, R. M.; Nelson, M. L.; Unice, K. M.; Keenan, J. J.; Paustenbach, D. J. Food Chem. Toxicol. 2013, 56, 60.

[6] Bu, X.-L.; He, B.-B.; Weng, J.-Y.; Jiang, C.-C.; Zhao, Y.-L.; Li, S.-M.; Xu, J.; Xu, M.-J. ACS Synth. Biol. 2020, 9, 2282.

[7] (a) Zhou, L.-J.; Li, S.-L.; Xu, B.; Ji, D.-T.; Wu, L.-Z.; Liu, Y.; Zhang, Z.-M.; Zhang, J.-L. Angew.Chem., Int. Ed. 2020, 59, 2769.

(b) Li, J.-X.; Xue, F.-L.; Tan, Y.-H.; Luo, S.-H.; Wang, Z.-Y. Acta Chim. Sinica 2011, 69, 1688 (in Chinese).

(李建晓，薛福玲，谭越河，罗时荷，汪朝阳，化学学报, 2011, 69, 1688.)

[8] (a) Yang, K.; Qiu, Y.-T.; Li, Z.; Wang, Z.-Y.; Jiang, S. J. Org. Chem. 2011, 76, 3151.

(b) Yang, K.; Li, Z.; Wang, Z.-Y.; Yao, Z.-Y.; Jiang, S. Org. Lett. 
2011, 13, 4340 .

(c) Marina, N.; Lanterna, A. E.; Scaiano, J. C. ACS Catal. 2018, 8, 7593.

[9] Dorel, R.; Grugel, C. P.; Haydl, A. M. Angew. Chem., Int. Ed. 2019, $58,17118$.

[10] (a) Zhao, H.-B.; Fu, H.; Qiao, R.-Z. J. Org. Chem. 2010, 75, 3311.

(b) Kadu, R.; Batabyal, M.; Kadyan, H.; Koner, A. L.; Kumar, S. Dalton Trans. 2019, 48, 7249.

(c) Li, J.-X.; Wang, Z.-Y.; Xue, F.-L.; Luo, S.-H. Acta Chim. Sinica 2011, 69, 2835 (in Chinese)

(李建晓, 汪朝阳, 薛福玲, 罗时荷, 化学学报, 2011, 69, 2835.)

[11] (a) Xu, Y.-M.; Chen, X.-Q.; Gao, Y.-Q.; Yan, Z.-C.; Wan, C.-F.; Liu, J.-B.; Wang, Z.-Y. J. Org. Chem. 2020, 85, 4354.

(b) Liu, Y.-X.; Xu, W.-Q.; Wang, X. Org. Lett. 2020, 12, 1448.

[12] (a) Yang, K.; Yao, C.; Gao, J.-J.; Chen, S.-H.; Zheng, X.-J.; Deng, L.-X.; Zheng, Y.-N.; Liu, M.-J.; Wang, Z.-Y. Chin. J. Org. Chem. 2020, 40, 4168 (in Chinese).

(杨凯, 姚辰, 高娟娟, 陈思鸿, 郑雪洁, 邓璐璇, 张毓娜, 刘美 娟, 汪朝阳, 有机化学, 2020, 40, 4168.)

(b) Sun, J.; Cao, J.; Han, Y.; Yan, C.-G. Chin. J. Org. Chem. 2020, 40, 4122 (in Chinese).

(孙晶, 曹釷, 韩莹, 颜朝国, 有机化学, 2020, 40, 4122.)

(c) Guo, W.; Zhao, M.-M.; Tan, W.; Zheng, L.-Y.; Tao, K.-L.; Fan, X.-L. Org. Chem. Front. 2019, 6, 2120.

(d) Chen, X.; Bai L.-L.; Zeng, W. Chin. J. Org. Chem. 2018, 38, 1859 (in Chinese).

(陈训, 白丽丽, 曾伟, 有机化学, 2018, 38, 1859.)

[13] (a) Balakumar, C.; Lamba, P.; Pran Kishore, D.; Lakshmi Narayana, B.; Venkat Rao, K.; Rajwinder, K.; Raghuram Rao, A.; Shireesha, B.; Narsaiah, B. Eur. J. Med. Chem. 2010, 45, 4904.

(b) Bouley, R.; Kumarasiri, M.; Peng, Z. H.; Otero, L. H.; Song, W.; Suckow, M. A.; Schroeder, V. A.; Wolter, W. R.; Lastochkin, E.; Antunes, N. T.; Pi, H. L.; Vakulenko, S.; Hermoso, J. A.; Chang, M.; Mobashery, S. J. Am. Chem. Soc. 2015, 137, 1738.

[14] Xu, W.; Jin, Y.-B.; Liu, H.-X.; Jiang, Y.-Y.; Fu, H. Org. Lett. 2011, $13,1274$.

[15] Kotipalli, T.; Kavala, V.; Janreddy, D.; Bandi, V.; Kuo, C.-W.; Yao, C.-F. Eur. J. Org. Chem. 2016, 2016, 1182.

[16] Modi, A.; Ali, W.; Mohanta, P. R.; Khatun, N.; Patel, B. K. ACS Sustainable Chem. Eng. 2015, 3, 2582.

[17] Yan, N.; You, C.-R.; Cai, M.-Z. J. Organomet. Chem. 2019, 897, 161.

[18] Sharma, R.; Vishwakarma, R. A.; Bharate, S. B. Adv. Synth. Catal. 2016, 358, 3027.

[19] Wang, L.-X.; Xiang, J.-F.; Tang, Y.-L. Eur. J. Org. Chem. 2014, $2014,2682$.

[20] Hu, B.-Q.; Wang, L.-X.; Xiang, J.-F.; Yang, L.; Tang, Y.-L. Chin. Chem. Lett. 2015, 26, 369

[21] Xu, W.; Fu, H. J. Org. Chem. 2011, 76, 3846.

[22] Kong, L.-L.; Fan, L.-Y. Chin. Chem. Lett. 2016, 27, 827.

[23] Nazarenko, K. G.; Shyrokaya, T. I.; Tolmachev, A. A. Synth. Commun. 2003, 33, 303.

[24] Zhou, J.; Fu, L.-B.; Lv, M.; Liu, J.-S.; Pei, D.-Q.; Ding, K. Synthesis 2008, 3974.

[25] Songsichan, T.; Promsuk, J.; Rukachaisirikul, V.; Kaeobamrung, J. Org. Biomol. Chem. 2014, 12, 4571.

[26] Tian, H.; Qiao, H.-W.; Zhu, C.-J.; Fu, H. RSC Adv. 2014, 4, 2694

[27] Xu, L.-T.; Jiang, Y.-W.; Ma, D.-W. Org. Lett. 2012, 14, 1150.

[28] Kumar, A.; Bishnoi, A. K. RSC Adv. 2014, 4, 41631.

[29] Iqbal, M. A.; Lu, L.; Mehmood, H.; Khan, D. M.; Hua, R. M. ACS Omega 2019, 4, 8207.

[30] Sayyad, N.; Cele, Z.; Aleti, R. R.; Bera, M.; Cherukupalli, S.; Chandrasekaran, B.; Kushwaha, N. D.; Karpoormath, R. Eur. J. Org. Chem. 2018, 2018, 5382.

[31] Yu, X.-Q.; Gao, L.-Q.; Jia, L.-A.; Yamamoto, Y.; Bao, M. J. Org. Chem. 2018, 83, 10352.

[32] Wang, F.; Zhao, P.; Xi, C.-J. Tetrahedron Lett. 2011, 52, 231.

[33] Yang, K.; Yang, J.-Q.; Luo, S.-H.; Mei, W.-J.; Lin, J.-Y.; Zhan,
J.-Q.; Wang, Z.-Y. Bioorg. Chem. 2021, 107, 104518.

[34] Krishna, M. H.; Thriveni, P. Heterocycl. Lett. 2018, 8, 229.

[35] Liu, Z.-B.; Zeng, L.-Y.; Li, C.; Yang, F.-B.; Qiu, F.-S.; Liu, S.-W.; Xi, B.-M. Molecules 2018, 23, 2325.

[36] Guo, S.-H.; Li, Y.; Tao, L.; Zhang, W.-W.; Fan, X.-S. RSC Adv. 2014, 4, 59289.

[37] Upadhyaya, K.; Thakur, R. K.; Shukla, S. K.; Tripathi, R. P. J. Org Chem. 2016, 81, 5046 .

[38] Li, T.; Chen, M.-L.; Yang, L.; Xiang, Z.-X.; Wang, Y.-W.; Li, F.; Chen, D.-Y. Tetrahedron 2016, 72, 868

[39] Shinde, M. H.; Kshirsagar, U. A. RSC Adv. 2016, 6, 52884.

[40] Nandwana, N. K.; Dhiman, S.; Saini, H. K.; Kumar, I.; Kumar, A. Eur. J. Org. Chem. 2017, 2017, 514.

[41] Dao, P.-D.-Q.; Cho, C.-S.; Ho, S.-L.; Sohn, H.-S. Curr. Org. Chem. 2018, 22, 85 .

[42] Xu, H.; Fu, H. Chem.-Eur. J., 2012, 18, 1180.

[43] Abe, T.; Takahashi, Y.; Matsubara, Y.; Yamada, K. Org. Chem. Front. 2017, 4, 2124

[44] Upadhyay, N. S.; Thorat, V. H.; Sato, R.; Annamalai, P.; Chuang, S C.; Cheng, C. H. Green Chem. 2017, 19, 3219.

[45] Weng, W.-Z.; Xie, J.; Zhang, B. Org. Biomol. Chem. 2018, 16, 3983.

[46] Liu, C.-C.; Parthasarathy, K.; Cheng, C.-H. Org. Lett. 2010, 12, 3518.

[47] Nohira, I.; Liu, S.; Bai, R.; Lan, Y.; Chatani, N. J. Am. Chem. Soc. 2020, 142, 17306

[48] de Salinas, S. M.; Sanjose-Orduna, J.; Odena, C.; Barranco, S.; Benet-Buchholz, J.; Perez-Temprano, M. H. Angew. Chem., Int. Ed. 2020, 59, 6239.

[49] Chary, R. G.; Dhananjaya, G.; Prasad, K. V.; Vaishaly, S.; Ganesh, Y. S. S.; Dulla, B.; Kumar, K. S.; Pal, M. Chem. Commun. 2014, $50,6797$.

[50] Zhao, H.; Wu, Y.-C.; Wei, T.; Cai, M.-Z. J. Chem. Res. 2014, 634.

[51] Wang, F.; Liu, H.-X.; Fu, H.; Jiang, Y.-Y.; Zhao, Y.-F. Org. Lett. 2009, 11, 2469.

[52] Huang, C.-Y.; Kavala, V.; Kuo, C.-W.; Konala, A.; Yang, T.-H.; Yao, C.-F. J. Org. Chem. 2017, 82, 1961

[53] Shi, Y.; Zhu, X.-B.; Mao, H.-B.; Hu, H.-W.; Zhu, C.-J.; Cheng, Y.-X. Chem.-Eur. J. 2013, 19, 11553

[54] Guastavino, J. F.; Barolo, S. M.; Rossi, R. A. Eur. J. Org. Chem 2006, 2006, 3898.

[55] Gong, X.-Q.; Li, G.-Q.; Gan, Z.-Y.; Yan, Q.-L.; Dou, X.-M.; Yang, D.-S. Asian J. Org. Chem. 2019, 8, 1472.

[56] Liu, Y.-Y.; Zeng, R.-S.; Pan, J.; Zou, J.-P. Chin. J. Chem. 2014, 32, 883.

[57] Lu, J.-Y.; Gong, X.-Y.; Yang, H.-J.; Fu, H. Chem. Commun. 2010 , $46,4172$.

[58] Liu, T.; Wang, R.-J.; Yang, H.-J.; Fu, H. Chem.-Eur. J., 2011, 17, 6765 .

[59] Kavala, V.; Yang, Z.-H.; Konala, A.; Huang, C.-Y.; Kuo, C.-W.; Yao, C.-F. J. Org. Chem. 2017, 82, 7280

[60] Cong, W.; Xu, J.-Y.; Yao, H.-Q. Chin. J. Org. Chem. 2014, 34, 1966. (丛蔚, 徐进宜, 姚和权, 有机化学, 2014, 34, 1966.)

[61] Dao, P. D. Q.; Lim, H. J.; Cho, C. S. ACS Omega 2018, 3, 12114.

[62] Ferraccioli, R.; Carenzi, D.; Rombola, O.; Catellani, M. Org. Lett. 2004, 6, 4759.

[63] Yadav, L.; Tiwari, M. K.; Shyamlal, B. R. K.; Chaudhary, S. J. Org. Chem. 2020, 85, 8121

[64] Bhakuni, B. S.; Kumar, A.; Balkrishna, S. J.; Sheikh, J. A.; Konar, S.; Kumar, S. Org. Lett. 2012, 14, 2838.

[65] Nishiyama, Y.; Nakamura, M.; Misawa, T.; Nakagomi, M.; Makishima, M.; Ishikawa, M.; Hashimoto, Y. Bioorg. Med. Chem. 2014, 22, 2799.

[66] Hu, Q.-F.; Gao, T.-T.; Shi, Y.-J.; Lei, Q.; Yu, L.-T. RSC Adv. 2018 8,13879 .

[67] Furuta, T.; Kitamura, Y.; Hashimoto, A.; Fujii, S.; Tanaka, K.; Kan, T. Org. Lett. 2007, 9, 183. 
[68] Liu, H.-L.; Han, W.-B.; Li, C.; Ma, Z.-Y.; Li, R.-X.; Zheng, X.-L.; Fu, H.-Y.; Chen, H. Eur. J. Org. Chem. 2016, 2016, 389.

[69] Chen, X.-W.; Liu, Y.-Y. ChemistrySelect 2018, 3, 7763.

[70] Donati, L.; Leproux, P.; Prost, E.; Michel, S.; Tillequin, F.; Gandon, V.; Poree, F. H. Chem.-Eur. J. 2011, 17, 12809.

[71] Chavan, S. P.; Bhanage, B. M. Eur. J. Org. Chem. 2015, 2015, 2405.

[72] Wang, Y.-Y.; Zhou, Y.; Lei, M.; Hou, J.-J.; Jin, Q.-H.; Guo, D.; Wu, W.-Y. Tetrahedron 2019, 75, 1180.

[73] De, R. P.; Bera, M. K. Synth. Commun. 2020, 50, 1780.

[74] Sarkar, S.; Dutta, S.; Dey, R.; Naskar, S. Tetrahedron Lett. 2012, 53, 6789.

[75] Peytam, F.; Adib, M.; Mahernia, S.; Rahmanian-Jazi, M.; Jahani, M.; Masoudi, B.; Mandavi, M.; Amanlou, M. Bioorg. Chem. 2019, $87,1$.

[76] Yang, P.; You, S.-L. Org. Lett. 2018, 20, 7684.

[77] Li, L.; Wang, M.; Zhang, X.-J.; Jiang, Y.-W.; Ma, D.-W. Org. Lett. 2009, 11, 1309

[78] Pan, J.; Xu, Z.; Zeng, R.-S.; Zou, J.-P. Chin. J. Chem. 2013, 31, 1022.

[79] Zhang, L.; Zhang, Y.-L.; Wang, X.; Shen, J.-K. Molecules 2013, 18 , 654.

[80] Hazarika, D.; Phukan, P. ChemistrySelect 2018, 3, 2474.

[81] Munoz, S. B.; Aloia, A. N.; Moore, A. K.; Papp, A.; Mathew, T.; Fustero, S.; Olah, G. A.; Prakash, G. K. S. Org. Biomol. Chem. 2016, 14, 85 .

[82] Gogoi, A.; Guin, S.; Rout, S. K.; Majji, G.; Patel, B. K. RSC Adv. 2014, 4, 59902.

[83] Sarkar, S.; Chatterjee, N.; Roy, M.; Pal, R.; Sarkar, S.; Sen, A. K. RSC Adv. 2014, 4, 7024

[84] Sarkar, S.; Pal, R.; Sen, A. K. Tetrahedron Lett. 2013, 54, 4273.

[85] Chatterjee, N.; Sarkar, S.; Pal, R.; Sen, A. K. Tetrahedron Lett. 2013, 54, 3748 .

[86] Hellal, M.; Cuny, G. D. Tetrahedron Lett. 2011, 52, 5508.

[87] Xiong, J.; Zhong, G.-F.; Liu, Y.-Y. Adv. Synth. Catal. 2019, 361, 550.

[88] Pacula, A. J.; Kaczor, K. B.; Wojtowicz, A.; Antosiewicz, J.; Janecka, A.; Dlugosz, A.; Janecki, T.; Scianowski, J. Bioorg. Med. Chem. 2017, 25, 126.

[89] Li, T.; Yang, L.; Ni, K.-D.; Shi, Z.-Y.; Li, F.; Chen, D.-Y. Org. Biomol. Chem. 2016, 14, 6297.
[90] Cao, L.; Luo, S.-H.; Wu, H.-Q.; Chen, L.-Q.; Jiang, K.; Hao, Z.-F.; Wang, Z.-Y. Adv. Synth. Catal. 2017, 359, 2961.

[91] Krasikova, V.; Katkevics, M. Chem. Heterocycl. Compd. 2013, 48, 1684.

[92] Balkrishna, S. J.; Bhakuni, B. S.; Chopra, D.; Kumar, S. Org. Lett. 2010, 12, 5394.

[93] Bhakuni, B. S.; Balkrishna, S. J.; Kumar, A.; Kumar, S. Tetrahedron Lett. 2012, 53, 1354.

[94] Pacula, A. J.; Scianowski, J.; Aleksandrzak, K. B. RSC Adv. 2014, 4, 48959.

[95] Wu, H.-Q.; Yang, K.; Luo, S.-H.; Wu, X.-Y.; Wang, N.; Chen, S.-H.; Wang, Z.-Y. Eur. J. Org. Chem. 2019, 2019, 4572.

[96] Wu, H.-Q.; Yang, K.; Chen, X.-Y.; Mani, A.; Wang, N.; Chen, S.-H.; Wang, Z.-Y. Green Chem. 2019, 21, 3782.

[97] Wang, F.; Chen, C.; Deng, G.; Xi, C.-J. J. Org. Chem, 2012, 77, 4148.

[98] Thanna, S.; Goins, C. M.; Knudson, S. E.; Slayden, R. A.; Ronning, D. R.; Sucheck, S. J. J. Org. Chem. 2017, 82, 3844.

[99] Chen, X.-W.; Hao, W.-Y.; Liu, Y.-Y. Org. Biomol. Chem. 2017, 15, 3423.

[100] Cai, S.-J.; Wang, F.; Xi, C.-J. Synthesis 2012, 44, 1892.

[101] Wang, X.-W.; Wu, C.-L.; Sun, Y.-W.; Yao, X.-Q. Tetrahedron Lett. 2017, 58, 3164.

[102] Kavala, V.; Wang, C.-C.; Barange, D. K.; Kuo, C.-W.; Lei, P.-M.; Yao, C.-F. J. Org. Chem. 2012, 77, 5022.

[103] Chen, Q.; Wang, Y.-P.; Hua, R.-M. Molecule 2019, 24, 3773.

[104] Gawande, S. D.; Kavala, V.; Zanwar, M. R.; Kuo, C.-W.; Huang, W.-C.; Kuo, T.-S.; Huang, H. N.; He, C. H.; Yao, C. F. Adv. Synth Catal. 2014, 356, 2599.

[105] Chen, Y.-Y.; Peng, Q.-J.; Zhang, R.; Hu, J.; Zhou, Y.-J.; Xu, L.-T.; Pan, X.-H. Synlett 2017, 28, 1201.

[106] Liu, S.-H.; Hu, Y.; Qian, P.-F.; Hu, Y.-W.; Ao, G.-Z.; Chen, S.-H.; Zhang, S.-L.; Zhang, Y.-N. Tetrahedron Lett. 2015, 56, 2211.

[107] Kitching, M. O.; Hurst, T. E.; Snieckus, V. Angew. Chem., Int. Ed. 2012, 51, 2925.

[108] Zhou, W.-J.; Jiang, Y.-X.; Chen, L.; Liu, K.-X.; Yu, D.-G. Chin. J. Org. Chem. 2020, 40, 3697 (in Chinese).

(周文俊, 蒋元旭, 陈亮, 刘开兴, 余达刚, 有机化学, 2020, 40, 3697.)

[109] Waldvogel, S. R.; Lips, S.; Selt, M.; Riehl, B.; Kampf, C. J. Chem. Rev. 2018, 118,6706

(Zhao, C.) 